\title{
Psicologia comunitária: relato de experiência de intervenção com usuários de álcool na atenção primária à saúde
}

Community psychology: intervention experience report with alcohol users in primary health care

Psicología comunitaria: informe de experiencia de intervención con usuarios de alcohol en atención primaria de salud

Karoline Giele Martins de Aguiar ${ }^{1 *}$, Lívia Araújo Sousa ${ }^{1}$, Rodrigo Santos Silva ${ }^{1}$, Jeffeson Jesus Santos ${ }^{1}$, Maria de Nazaré Lima Margalho².

\section{RESUMO}

Objetivo: Relatar a experiência vivenciada por uma profissional de psicologia de um serviço de alta complexidade em parceira com a atenção primária em saúde, com uma população usuária de álcool, em uma cidade do interior do Maranhão. Métodos: Trata-se de um relato de experiência, utilizando-se como referência a psicologia social comunitária, foram realizados três encontros no território, com a participação de 22 indivíduos e membros a equipe da Unidade Básica de Saúde - UBS. Resultados: Observou-se a adesão dos indivíduos nos encontros, aproximação com os serviços de saúde e constatação da demanda de escuta qualificada por parte dessa população, que muitas vezes são estigmatizados devido o uso de álcool. Considerações Finais: Conclui-se que a psicologia social comunitária como uma possibilidade de intervenção na promoção a saúde, prevenção e intervenção junto a população usuária de álcool e outras drogas.

Palavras-chave: Psicologia social, Álcool, Atenção primária à saúde.

\begin{abstract}
Objective: To report the experience of a psychology professional in a highly complex service in partnership with primary health care, with an alcohol user population, in a city in the interior of Maranhão. Methods: This is an experience report, using community social psychology as a reference, three meetings were held in the territory, with the participation of 22 individuals and members of the Basic Health Unit - UBS team. Results: Individuals adhered to the meetings, approached the health services and found the demand for qualified listening by this population, who are often stigmatized due to the use of alcohol. Final Considerations: It is concluded that community social psychology as a possibility of intervention in health promotion, prevention and intervention with the population using alcohol and other drugs.
\end{abstract}

Keyword: Alcohol, Social psychology, Primary health care.

\section{RESUMEN}

Objetivo: Informar la experiencia de un profesional de psicología en un servicio altamente complejo en asociación con la atención primaria de salud, con una población de consumidores de alcohol, en una ciudad en el interior de Maranhão. Métodos: Este es un informe de experiencia, utilizando la psicología social

\footnotetext{
1Universidade Ceuma (CEUMA). Imperatriz - MA. *E-mail: karol.giele@hotmail.com

${ }^{2}$ Programa Estratégia Saúde da Família. Imperatriz - MA
} 
comunitaria como referencia. Se realizaron tres reuniones en el territorio, con la participación de 22 individuos y miembros de la Unidad de Salud Básica - equipo de UBS. Resultados: las personas se adhirieron a las reuniones, se acercaron a los servicios de salud y encontraron la demanda de una audiencia calificada por parte de esta población, que a menudo se estigmatiza debido al uso de alcohol. Consideraciones finales: Se concluye que la psicología social comunitaria como una posibilidad de intervención en la promoción de la salud, prevención e intervención con la población que consume alcohol y otras drogas.

Palabra clave: Alcohol, Psicología social, Atención primaria de salud.

\section{INTRODUÇÃO}

Ao longo da história, o ser humano recorreu ao uso de substâncias como álcool, tabaco e outras drogas, seja em rituais religiosos, para se alienar do sofrimento, ou na busca do prazer. As circunstâncias, as motivações e as novas formas de obtenção das substâncias psicoativas variaram bastante ao longo dos tempos, assumindo características próprias de acordo com cada época e cada contexto social em que está inserida, levando a circunstâncias distintas de vulnerabilidade individual, social e comunitária. (BRASIL, 2015).

Segundo a Organização Mundial de Saúde (OMS, 2015), droga é a toda a substância que, introduzida no organismo vivo, modifica uma ou mais das suas funções, independentemente de ser lícita ou ilícita. O uso de drogas na atualidade é uma preocupação mundial (BRASIL, 2019). Entre 2000 e 2015, houve um crescimento de $60 \%$ no número de mortes causadas diretamente pelo uso de drogas, sendo este dado o recorte de apenas uma das consequências do problema. Tal condição extrapola as questões individuais e se constitui como um grave problema de saúde pública, com reflexos nos diversos segmentos da sociedade (BRASIL,2019; UNODC, 2018.). De acordo com o III Levantamento Nacional sobre o uso de drogas pela população brasileira, 2,3 milhões de pessoas entre 12 e 65 anos apresentaram dependência de álcool (BASTOS et al., 2017). A dependência por uso de álcool tem maior frequência entre os homens, maiores de 18 anos e com baixo nível de escolaridade (BASTOS et al., 2017).

Entre a população adolescente (12-17 anos), observou-se que a prevalência da dependência em indivíduos que fizeram uso de álcool nos últimos 12 meses $(2,6 \%)$ foi maior que na população geral $(0,6 \%)$ dessa faixa etária, o que pode indicar "uma chance maior de dependência entre os indivíduos que iniciam o consumo de álcool mais precocemente" (BASTOS et al., 2017, p. 128). Em relação à localidade geográfica, observou-se que há maior prevalência de dependência de álcool na Região Norte $(5,1 \%)$, seguida pela Região Nordeste $(4,8 \%)$, e menor prevalência na Região Sul (1,5\%) (BASTOS et al., 2017).

Conforme aponta pesquisas realizadas em dois Centro de Atenção Psicossocial - Álcool e outras Drogas (CAPS Ad III), localizados em municípios do interior do estado do Maranhão, o perfil dos usuários de drogas em tratamento é semelhante ao encontrado em outros estudos nacionais. A maioria dos usuários de drogas na região são adultos jovens, homens, com idade média de 30 anos e solteiros, possuem, prevalece a baixa escolaridade (AGUIAR KG, et al., 2019; CARVALHO IA, et al., 2020).

Observou-se, ainda, a presença de comorbidades psiquiátricas, com alguns pacientes relatando a presença de alucinações, ansiedade, delírios, alteração de humor, agitação e tremores (CARVALHO IA, et al., 2020). Ademais, a falta de uma rede de apoio social, como a família, mostrou-se como fator de risco associado ao uso abusivo de substâncias químicas, com pacientes relatando possuírem atritos familiares devido ao uso dessas substâncias (AGUIAR KG, et al., 2019; CARVALHO IA, et al., 2020).

O acolhimento e tratamento as pessoas usuárias de álcool, de acordo com a portaria GM/MS № 336, 16 de fevereiro de 2000, é feito nos Centros de Atenção Psicossocial (CAPS). Essas unidades, são constituídas por uma equipe multiprofissional que atua tanto no atendimento a situações de crise como nos processos de reabilitação psicossocial. Essa portaria determina que a equipe multiprofissional pode ser composta basicamente por psiquiatra, psicólogo, enfermeiro e assistente social, conforme portaria $n^{\circ} 130$, de 26 de janeiro de 2012 (BRASLI, 2012). 
Caso não haja nenhum CAPS no município ou o uso não se configure quadro de dependência química o atendimento de saúde mental deve ser feito nas Unidades Básicas de Saúde (UBS), por um psicólogo da UBS ou por um psicólogo do NASF (Núcleo de Apoio à Saúde da Família) dentro da Unidade de saúde, podendo ser feita também visitas domiciliares aos pacientes, como determina a Política Nacional de Atenção Básicas (PNAB) e a Portaria № 3.088, de 23 de dezembro de 2011 . Além disso, outros locais que oferecem serviços voltados ao atendimento dessas pessoas são as Unidades de Acolhimento (UAs), destinadas à residência transitória de pessoas em situação de vulnerabilidade que possam ou não está associadas ao uso de álcool e outras drogas, além de Unidades de Referência Especializada em Hospital Geral (BRASIL, 2011).

Nesse cenário, a Psicologia na Comunidade atua com o intuito de aproximar-se das pessoas e dos grupos sociais, visando uma melhoria na qualidade de vida dos indivíduos e da população, através da utilização dos conhecimentos psicológicos (ANDERY AA, 2012). Essa aproximação revela um distanciamento do lugar tradicional de se fazer Psicologia, isto é, a clínica, ou a indústria (ANDERY AA, 2012). Nesse sentido, a Psicologia na Comunidade caracteriza-se como "um movimento de aproximação do cotidiano das pessoas, principalmente nos bairros e instituições populares onde a grande parcela da população vive" (ANDERY AA, 2012, p. 203).

Assim sendo, alguns estudos demonstram essa prática, seja ao disponibilizar um serviço de plantão psicológico em um local religiosos, atendendo a demandas de uma escuta ativa e apoio emocional (SCORSOLINI-COMIN, 2014); um plantão comunitário em uma comunidade de baixa renda, através de atendimento grupal e trocas de experiências entre os participantes (COIN-CARVALHO JE e OSTRONOFF $\mathrm{VH}, 2014$ ); ou, ainda, a criação de espaços para que jovens da comunidade possam ter trocar de experiências e criassem sentimento de pertença, de reconhecimento e de apoio social (SCISLESKI AC, et al., 2006). Campos RH (2015) destaca que o papel do psicólogo comunitário junto à população é desenvolver métodos e processos de conscientização para que os grupos e os sujeitos sociais, ao tornarem-se cientes da sua realidade sócio-cultural, assumam seu papel enquanto indivíduos detentores de sua própria história, visando assim, alcançar consciência crítica e autonomia.

Nesse sentido, em um estudo qualitativo, Giffoni FA e Santos MA (2011) analisam as potencialidades da Terapia Comunitária (TC) como recurso para o enfrentamento do uso abusivo de álcool. Como resultado, constataram que a TC proporciona ao participante o desenvolvimento de competências sociais, como capacidade de liderança e autonomia, assim como gera reflexão crítica sobre o papel do uso abusivo de álcool na sua vida e as consequências deste no contexto social. As essas mudanças perceptuais são possíveis devido à rede de apoio social que se estabelece na TC, através das trocas de experiências entre os participantes e do ambiente empático e acolhedor gerado (GIFFONI FA e SANTOS MA, 2011),

Ademais, Aguiar KG, et al. (2019) sinalizam para o impacto que a rede de apoio social exerce na manutenção da abstinência. Assunção et al. (2019) corroboram essa visão, ao relatarem que a criação de uma relação terapêutica empática contribuiu para a adesão e o tratamento de grupos de usuários de drogas. Além disso, observou-se um caráter emancipatório em suas práticas, ao indagarem esses sujeitos acerca dos riscos e danos a que poderiam estar expostos devido ao uso abusivo e prejudicial de drogas, permitindo que estes refletissem criticamente sobre os seus hábitos (ASSUNÇÃO Jl, et al., 2019). Com isso, destacam o caráter emancipatório das reflexões, sempre evitando um discurso moralista e invasivo, permitindo que esses indivíduos se colocassem num local de protagonismo de suas vidas e de suas escolhas, aos quais as drogas ocupavam um lugar central, gerando prejuízos nas relações sociais, familiares e laborais. (ASSUNÇÃO JI, et al., 2019).

\section{MÉTODOS}

Trata-se de um relato de experiência, de abordagem reflexiva, acerca da vivência do profissional de psicologia em uma perspectiva da psicologia social comunitária. A atividade foi idealizada pela enfermeira da Estratégia de Saúde a Família (ESF), em parceria com a psicóloga do CAPS ADIII, no munícipio de ImperatrizMA. Foram realizados 03 (três) encontros, com frequência quinzenal no território de abrangência da equipe 
de ESF, o primeiro momento ocorreu em um bar, disponibilizado pela proprietária, o segundo momento foi realizado dentro da Unidade Básica de Saúde -UBS e o terceiro encontro foi na Associação de Moradores do Bairro. As atividades contaram com a participação de 22 indivíduos com hipótese de quadro de alcoolismo e com 4 Agentes Comunitários de Saúde- ACS, que indicaram os indivíduos para a participação no projeto, a enfermeira da ESF e a psicóloga do CAPS Ad III.

No primeiro momento foi realizado a apresentação do grupo e realizado psicoeducação abordando o uso de drogas, com ênfase no uso de álcool, no segundo encontro foi realizado a aplicação do rastreador para o uso de drogas (ALCOHOL, SMOKING AND SUBSTANCE INVOLVEMENT SCREENING TEST) - ASSIST, desenvolvido pela OMS, e validado para a população brasileira (HENRIQUE IFC, et al., 2004), e no último encontro foi realizado roda de conversa, com a temática "o cuidar do homem e suas implicações na condução de sua saúde". As atividades seguiram uma dinâmica sistemática, no entanto as demandas trazidas pelos indivíduos foram acolhidas, e obtiveram as devidas orientações, alguns casos houve discussão após o encerramento para melhor atender as demandas de saúde.

\section{RESULTADOS}

As atividades foram desenvolvidas pelo profissional de psicologia, após o diálogo com a enfermeira da UBS, que indicou que no território tem alto índice de indivíduos que fazem uso nocivo de álcool. Então estabeleceu-se uma intervenção breve, que proporcionasse efetividade nos resultados e adesão ao tratamento com essa população.Os indivíduos foram convidados pelas ACS, alguns estavam sob efeito do uso de álcool, não foi realizado avaliação para a constatação, apresentavam sinais como odor do álcool e isso indica que a ação in loco aproximou os indivíduos das práticas em saúde. Após a aplicação do rastreador para uso nocivo de álcool, os resultados indicaram que todos os 22 indivíduos necessitavam de intervenção especializada, o instrumento impactou os indivíduos, a partir do resultado, demostraram o interesse em recorrer ao serviço especializado de saúde, por demanda espontânea.

A adesão e aproximação dos indivíduos com os profissionais resultaram na roda de conversa, no qual o principal eixo temático era a autonomia do homem e suas implicações na saúde, nesse momento para além da saúde, os participantes relataram as fragilidades dos vínculos familiares e comentários, estraçalhados pelo uso de álcool, observou-se a necessidade de espaço de escuta e compreensão no qual a fala era permitida sem julgamentos.

\section{DISCUSSÃO}

As atividades realizadas foram dialogadas e organizadas de acordo com a demanda apresentada pelo território, visando a integração do saber cientifico com a prática comunitária, que também possibilita estratégias de prevenção, compreendendo o indivíduo no seu espaço comunitário e dentro de seus grupos sociais (DA LUZ AA e PERES EL, 1997; FERNANDES VM e ROMANINI M, 2016). Os indivíduos foram convidados para os encontros pelos ACS, com isso destaca-se a inclusão desses indivíduos no território, a aproximação dos serviços de intervenção in loco, oportunizou a aderência nos encontros, corroborando a efetividade da psicologia comunitária, no qual os indivíduos estão comprometidos com a própria transformação (FERNANDES VM, ROMANINI M, 2016).

A inserção da (o) psicóloga (o) no território, possibilita a oportunidade de escuta das necessidades da comunidade no que tange suas reais expectativas em relação a aproximação dos serviços de alto complexidade, respeitando a autonomia do indivíduo (FREITAS MF, 1998). A adesão dos indivíduos e a participação de forma ativa nos três momentos, se tornou possível no momento que compreenderam a relevância da autonomia do próprio cuidar, associado ao processo de psicoeducação sobre os efeitos nocivos ocasionados pelo uso de álcool. A prática de psicoeducação como forma de promoção e prevenção, ao uso de drogas tem evidenciado intervenções efetivas, auxiliando na diminuição do quadro de ansiedades de usuários de drogas (FATIMA GC, 2019). 
A participação comunitária proporcionou o cuidado em saúde em sua integralidade, as demandas foram absorvidas pela equipe e direcionadas, indicando o espaço de fala e escuta qualificada do sofrimento presente, das vulnerabilidades sociais e fragilidade dos vínculos afetivos, em decorrência do uso de álcool. Estudo aponta a relação entre a fragilidade dos vínculos familiares associados ao uso de álcool (RAMOS, FS, 2019). A ação comunitária abrange a realidade do indivíduo dentro de seu contexto familiar e sua relação com a comunidade, com isso intensifica a importância da inserção de ações junto a atenção primária na prevenção e na intervenção, visando fortalecer as equipes e as comunidades, diminuindo os estigmas do uso de álcool (PAIVA FS, et al,. 2012).

\section{CONSIDERAÇÕES FINAIS}

Considerando a complexidade do uso de drogas, incluindo o álcool, droga lícita que permeia diversos contextos sociais, fazendo parte de contexto histórico e de rituais em diversas culturas. O desafio torna-se maior, sendo a prática da psicologia comunitária uma possibilidade de intervenção, aproximando os indivíduos aos serviços de saúde. As limitações do trabalho se deram pela limitação do número de participantes, no entanto abre as possibilidades de reflexão da atuação do profissional de psicologia em ações comunitárias, extra muros, aproximando a prática profissional junto as comunidades. Sugere-se reflexão sobre as práticas profissionais, associadas as intervenções junto à atenção básica como forma de promoção a saúde e prevenção ao uso de drogas nos territórios.

\section{REFERÊNCIAS}

1. AGUIAR, Karoline Giele Martins; MELLO, Luana Thereza Nesi de; ANDRETTA, Ilana. Usuários de crack maranhenses: habilidades sociais, habilidades de enfrentamento e suporte social. Revista Psicologia em Pesquisa, [s.I.], v. 13, n. 2, p. 81-106, 6 nov. 2019. Universidade Federal de Juiz de Fora. http://dx.doi.org/10.34019/19821247.2019.v13.25805.

2. ANDERY, Alberto Abib. Psicologia na comunidade. In: LANE, Silvia T. M.; CODO, Wanderley (orgs.). Psicologia Social: o homem em movimento. 14. ed. São Paulo: Editora Brasiliense, 2012.

3. ASSUNÇÃO, Júlia Inácia Vieira et al. Eiras e Beiras: atenção psicossocial a pessoas com problemas decorrentes do uso de drogas. Psicologia \& Sociedade, [s.I.], v. 31, e178671, p. 1-11, 2019. http://dx.doi.org/10.1590/18070310/2019v31178671.

4. BASTOS, Francisco Inácio Pinkusfeld Monteiro et al. (Org.). III Levantamento Nacional sobre o uso de drogas pela população brasileira. Rio de Janeiro: FIOCRUZ/ICICT, 2017.

5. BRASIL. Decreto $n^{\circ}$ 9.761, de 11 de abril de 2019. Diário Oficial [da] União, Poder Executivo, Brasília, DF, 11 abril. 2019. Seção 1, p. 7.

6. BRASIL. Portaria, de 26 de janeiro de 2012. Redefine o Centro de Atenção Psicossocial de Álcool e outras Drogas $24 \mathrm{~h}$ (CAPS AD III) e os respectivos incentivos financeiros.

7. BRASIL. Ministério da Saúde. Departamento de Ações Programáticas Estratégicas (ed.). Guia estratégico para o cuidado de pessoas com necessidades relacionadas ao consumo de álcool e outras drogas: Guia AD. Brasília: Ministério da Saúde, 2015.

8. BRASIL. Portaria № 3.088, de 23 de dezembro de 2011. Institui a Rede de Atenção Psicossocial para pessoas com sofrimento ou transtorno mental e com necessidades decorrentes do uso de crack, álcool e outras drogas, no âmbito do Sistema Único de Saúde (SUS).

9. CAMPOS, Regina Helena de Freitas. Introdução: a psicologia social comunitária. In: CAMPOS, Regina Helena de Freitas (org.). Psicologia social comunitária: da solidariedade à autonomia. 20. ed. Petrópolis, RJ: Editora Vozes, 2015.

10. CARVALHO, I. A. B.; MENEZES, K. S.; MAGALHÃES, J. M.; AMORIM, F. C. M.; FERNANDES, M. A.; CARVALHO, C. M. M. S Perfil dos usuários de substâncias psicoativas. Rev. Fun. Care Online. 2020 jan/dez; 12:325-330. http://dx.doi.org/ 10.9789/2175-5361.rpcfo.v12.7095.

11. COIN-CARVALHO, João Eduardo; OSTRONOFF, Vera Helena. Cuidado e transformação social: avaliação da implantação do plantão comunitário no complexo da funerária.: avaliação da implantação do plantão comunitário no Complexo da Funerária. Estudos de Psicologia, Natal, v. 19, n. 2, p. 138-144, 2014.

12. DA LUZ, Araci Asinelli; PERES, Emerson Luiz. Reflexões sobre a extensão universitária e a participação da psicologia num programa de prevenção ao abuso de álcool e outras drogas. Interação em Psicologia, v. 1, n. 1, 1997.

13. DE FÁtIMA GUARIENTO, C., et al. Prevenção e Promoção de Saúde no CAPS AD através de oficinas de psicoeducação. Revista Eletrônica Científica Da UERGS, v. 5, n. 2, p. 191-197, 2019. 
14. FERNANDES, Vitória Merten; ROMANINI, Moises. O CAPS AD III e sua relação com a comunidade:(re) pensando os processos de desinstitucionalização e reabilitação psicossocial. CRIAR E OUSAR, 2016.

15. FREITAS, Maria de Fatima Quintal de. Inserção na comunidade e análise de necessidades: reflexões sobre a prática do psicólogo. Psicologia: reflexão e crítica, v. 11, n. 1, p. 175-189, 1998.

16. GIFFONI, Francinete Alves de Oliveira; SANTOS, Manoel Antônio dos. Terapia comunitária como recurso de abordagem do problema do abuso do álcool, na atenção primária. Revista Latino-Americana de Enfermagem, [s.I.], v. 19, n. spe, p. 821-830, jun. 2011. http://dx.doi.org/10.1590/s0104-11692011000700021.

17. HENRIQUE, IFS., et al. Validação da versão brasileira do teste de triagem do envolvimento com álcool, cigarro e outras substâncias (ASSIST). Revista da Associação Médica Brasileira, 2004; 50(2), 199-206.

18. Ministério da Saúde. Portaria GM/MS no 336, de 19 de fevereiro de 2002. Estabelece que os Centros de Atenção Psicossocial poderão constituir-se nas seguintes modalidades de serviços: CAPS I, CAPS II e CAPS III, definidos por ordem crescente de porte/complexidade e abrangência populacional. Diário Oficial [da] União, Brasília, DF, 9 fev.2002b:

19. Ministério da Saúde. Portaria $n^{\circ} 2.436,21$ de setembro 2017. Institui o Plano Nacional de Atenção Básica. Diário Oficial [da] União, Brasília,DF,21set.2017b:

20. Mistério da Saúde. Portaria GM/MS no 3.088, de 23 de dezembro de 2011. Institui a Rede de Atenção Psicossocial para pessoas com sofrimento ou transtorno mental e com necessidades decorrentes do uso de crack, álcool e outras drogas, no âmbito do Sistema Único de Saúde (SUS). Diário Oficial [da] União, Brasília, DF, 23 dez. 2011b.

21. PAIVA, FS., et al. Fortalecendo redes sociais: desafios e possibilidade na prevenção ao uso de drogas na atenção primária à saúde fortalecendo redes sociais. Aletheia, n. 37, p. 57-72, 2012.

22. RAMOS, Flávia Silva. A relação entre o vínculo afetivo familiar e uso de álcool e drogas na infância e na adolescência. Caderno de Direito da Criança e do Adolescente, v. 1, p. 10-10, 2019.

23. SCISLESKI, Andrea Cristina Coelho; MARASCHIN, Cleci; TITTONI, Jaqueline. A psicologia social e o trabalho em comunidades: limites e possibilidades. Interamerican Journal of Psychology, Porto Alegre, v. 40, n. 1, p. 4754, abr. 2006.

24. UNODC. Relatório Mundial sobre Drogas 2018: crise de opioides, abuso de medicamentos sob prescrição; cocaína e ópio atingem níveis recordes. Disponível em: Acesso: 29 abr. 2019. 